\section{Videolaryngoskopie I: bei der Notfallintubation besser!}

\begin{abstract}
Die primären Erfolgsraten der direkten Laryngoskopie bei Notfallintubationen auf der Erwachsenenintensivstation liegen bei nur 50-60\%. Im Kindesalter sind die Zahlen sicher nicht besser. Kann die mittlerweile vielerorts etablierte Methode der Videolaryngoskopie (VL) nun endlich diese Rate zu verbessern helfen? Ja, sie kann!
\end{abstract}

$\mathrm{n}$ einer prospektiven randomisierten Studie, die auf einer internistischen 16-Betten-Intensivstation stattfand, wurde geprüft, ob Nicht-Anästhesisten mit dem VL Glidescope ${ }^{\odot}$ Notfallintubationen erfolgreicher meistern können. Es kam eine exakt formulierte Methodik zur Anwendung. 117 Notfallintubatio-

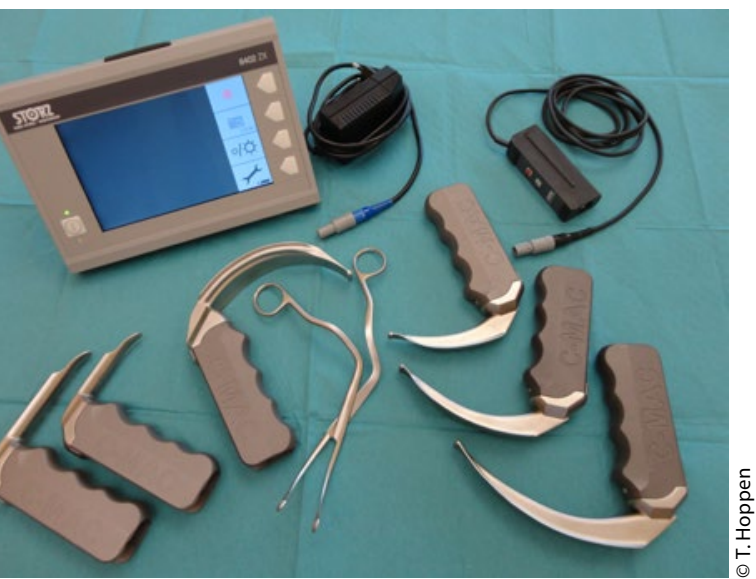

\section{Videolaryngoskopie II: nun auch beim Neugeborenen überlegen}

Erst kürzlich wurde der Videolaryngoskopie (VL) im Neugeborenenalter noch fehlende Evidenz attestiert [1]. Diese Cochrane-Aussage konnte nun durch die Veröffentlichung einer randomisierten Studie in Pediatrics eindrucksvoll entkräftet werden [2].

D ie Untersuchung von O'Shea und Kollegen wurde am Royal Women's Hospital in Melbourne, einer Einrichtung mit 7.500 Geburten pro Jahr, davon 300 Frühgeborene unter 1.500 g durchgeführt. 206 erstmalige orale Intubationsversuche im Kreißsaal beziehungsweise auf der neonatologischen Intensivstation wurden ausgewertet. Das medi- nen - meist durch Erstjahresassistenten durchgeführt - wurden ausgewertet.

Alle Patienten wurden erfolgreich intubiert. Mit dem VL gelang dies im ersten Versuch bei $74 \%$, mit der direkten Methode nur bei $40 \%$. Lediglich bei $9 \%$ aller VL-Intubationen waren mehr als zwei Versuche nötig, bei der direkten Methode bei $27 \%$. Bei der direkten Methode musste auf die VL gewechselt werden, andersherum jedoch nicht. Die durchschnittlichen Zeiten betrugen bei VL 120 Sekunden, direkt 218 Sekunden. Ösophageale Intubationen mit VL: 0\% und direkt: $7 \%$.

Die Studie wurde aufgrund der erheblichen Diskrepanz in den Ergebnissen

Abb. 1: Ein umfassendes Videolaryngoskopieset mit Spatelgrößen für alle Altersstufen. Die handliche PocketmonitorVersion ist ebenfalls sehr hilfreich, jedoch hier nicht mit abgebildet

aus Sicherheitsgründen vorzeitig beendet und auf der untersuchten Station die VL als Standard für die Notfallintubation eingeführt.

Silverberg MJ et al. Comparison of video laryngoscopy versus direct laryngoscopy during urgent endotracheal intubation: a randomized controlled trial. Crit Care Med 2015;43:636-41

\section{Kommentar}

Die VL ist nicht mehr primär dem schwierigen Atemweg vorbehalten. In der vorliegenden Studie wurde diese Patientengruppe von vornherein ausgeschlossen. Nein die VL (Abb. 1) ermöglicht neben dem Teaching, einer Foto- und/oder Videodokumentation, einer höheren Rechtssicherheit und einem kontrollierten beziehungsweise vorsichtigerem Vorgehen insbesondere in Bezug auf Prävention von Stimmbandläsionen auch eine höhere Trefferrate in zudem kürzerer Anwendungszeit als die direkte Methode. Fraglos liegen die primären Erfolgsraten der direkten Laryngoskopie unter kontrollierten Bedingungen, zum Beispiel bei einer elektiven Anästhesieeinleitung, bei über $90 \%$. Im Notfall sieht das aber anders aus: Allein aus Patientensicherheitsgründen sollte zumindest in dieser $\mathrm{Si}$ tuation die VL generell zum Standard werden und vorbereitend in Szenarien trainiert werden. Kinder haben ein noch längeres Leben vor sich als Erwachsene. Worauf warten wir also noch? Dr. Thomas Hoppen

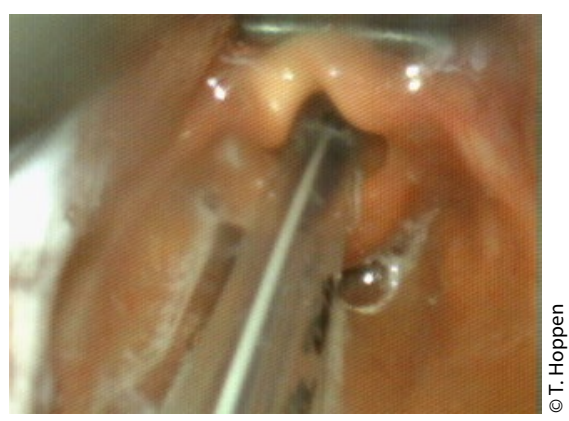

ane Alter der Frühgeborenen betrug 29 Schwangerschaftswochen, das Gewicht lag zwischen 816 und $1.884 \mathrm{~g}$.

Alle intubierenden Ärzte hatten maximal 6 Monate praktische Erfahrung in einer Level-I-Neonatologie. Es handelte sich dabei um insgesamt 36 Ärzte, darunter rund $50 \%$ mit $0-2$ und nur $7 \%$ mit $\geq 10$ erfolgreichen Intubationen im Zeit-
Abb. 1: Erfolgreiche VL-Intubation bei einem Frühgeborenen mit Surfactantmangel

raum vor Durchführung dieser Studie. Zur Anwendung kam ein modifiziertes traditionelles VL (LaryFlex, Acutronics, Hirzel, Switzerland). Der unmittelbare Intubationserfolg wurde kalorimetrisch 